\title{
杭州湾潮流中湍应力和拖曳系数 估算的三种方法
}

\author{
方国洪 李身锋*曹德明 \\ （中医科学院海洋研究所,青岛；＂华东师范大学河口海岸研究所，上海）
}

测量和估算湍应力的主要方法之一是利用潮波支配方程. 根据杭州湾的实际情况，我们 采用了三种方法，得到了较一致的结果. 第一种方法直接利用动量方程. 第二种方法是将动 量方程在一个海区上积分，得出应力项在全域上的总体估计值．第三种方法是利用能量方程， 得出拖电系数估计值. 本文所用的第一种方法是 Bowden 等 ${ }^{[1]}$ 及 Wolf ${ }^{[2]}$ 所用方法的一个直接 的推广; 第三种方法的应用最早可追溯到 $\mathrm{T}_{\text {aylor }}{ }^{[3]}$; 第二种方法则是这里首次提出的.

\section{一、理 论 依据}

1. 基本方程 计算所依据的潮波支配方程取为:

$$
\begin{gathered}
\frac{\partial\langle u\rangle_{z}}{\partial t}-f\langle v\rangle_{z}+\frac{\tau_{x b}}{h}+g \frac{\partial \zeta}{\partial x}=0, \frac{\partial\langle v\rangle_{z}}{\partial t}+f\langle u\rangle_{z}+\frac{\tau_{y b}}{h}+g \frac{\partial \zeta}{\partial y}=0, \\
\frac{\partial \zeta}{\partial t}+\frac{\partial\left(h\langle\dot{u}\rangle_{z}\right)}{\partial x}+\frac{\partial\left(h\langle v\rangle_{z}\right)}{\partial y}=0,
\end{gathered}
$$

其中 $t, x, y, z, u, v, \zeta, f, h, g$ 均为惯用符号; \langle\rangle$_{x}$ 表示从海底到海面取平均; $\tau_{x}, \tau_{y}$ 为切应 力在 $x, y$ 方向的分量, 下标 $b$ 表示在海底处值.

$$
\begin{gathered}
\text { 对方程(1)-(3)作运算 } \lim _{T \rightarrow \infty} \frac{1}{T} \int_{-T}^{T}() e^{i \sigma t} d t \text {, 则可得它们的广义 Fourier 变换式: } \\
\begin{array}{c}
-i \sigma\langle\tilde{u}\rangle_{z}-t\langle\tilde{v}\rangle_{x}+\frac{\tilde{\tau}_{x b}}{h}+g \frac{\partial \tilde{\zeta}}{\partial x}=0,-i \sigma\langle\tilde{v}\rangle_{z}+f\langle\tilde{u}\rangle_{x}+\frac{\tilde{\tau}_{y b}}{h}+g \frac{\partial \tilde{\zeta}}{\partial y}=0, \\
-i \sigma \tilde{\zeta}+\frac{\partial\left(h\langle\tilde{u}\rangle_{z}\right)}{\partial x}+\frac{\partial\left(h\langle\tilde{v}\rangle_{z}\right)}{\partial y}=0,
\end{array}
\end{gathered}
$$

上列诸式中符号 代表 Fourier 系数:

$$
\left(\tilde{u}, \tilde{v}, \tilde{\zeta}, \tilde{\tau}_{x}, \tilde{\tau}_{y}\right) \equiv \lim _{T \rightarrow \infty} \frac{1}{T} \int_{-T}^{T}\left(u, v, \zeta, \tau_{x}, \tau_{y}\right) e^{i \sigma t} d t .
$$

2. 方法 1 假定在相距 $L$ 的两地 $\mathrm{A}$ 和 $\mathrm{B}$ 已进行水位观测, 并在它们之间若干点进行了 潮流观测. 取新的坐标系 $\left(x^{\prime}, y^{\prime}, z\right)$ 使 $x^{\prime}$ 轴由 $\mathrm{A}$ 指向 $\mathrm{B}$, 相应的水平流速分量为 $u^{\prime}, v^{\prime}$, 则在 $x^{\prime}$ 方向从 $\mathrm{A}$ 到 $\mathrm{B}$ 对动量方程求平均, 可得

$$
\left\langle\tilde{\tau}_{x^{\prime} b}\right\rangle_{x^{\prime}} \approx\langle h\rangle_{x^{\prime}}\left\langle\frac{\tilde{\tau}_{x^{\prime} b}}{h}\right\rangle_{x^{\prime}}=\langle h\rangle_{x^{\prime}}\left\{i \sigma\left\langle\tilde{u}^{\prime}\right\rangle_{s x^{\prime}}+f\left\langle\tilde{v}^{\prime}\right\rangle_{x x^{\prime}}-\frac{g}{L}\left(\xi_{B}-\xi_{A}\right)\right\},
$$

式中〈的下标 $x^{\prime}$ 表示沿 $x^{\prime}$ 方向从 A 到 B 取平均. $y^{\prime}$ 方向的关系式类似可得.

本文 1985 年 1 月 18 日收到.

第 17 期 
3. 方法 2 如在海区 $\theta$ 上对方程 (4)和(5)积分,并应用 Green 公式,可得

$$
\begin{aligned}
& \left\langle\tau_{x b}\right\rangle_{\Omega} \approx\langle h\rangle_{\rho}\left\langle\frac{\tilde{\tau}_{x b}}{h}\right\rangle_{\Omega}=\langle h\rangle_{\rho}\left\{i \sigma\langle\tilde{u}\rangle_{x \Omega}+t\langle\tilde{v}\rangle_{x Q}-\frac{g}{\Omega} \oint_{\Gamma} \tilde{\zeta} d y\right\}, \\
& \left\langle\tau_{y b}\right\rangle_{\rho} \approx\langle h\rangle_{0}\left\langle\frac{\tilde{\tau}_{y b}}{h}\right\rangle_{e}=\langle h\rangle_{e}\left\{i \sigma\langle\tilde{v}\rangle_{s o}-f\langle\tilde{u}\rangle_{x o}-\frac{g}{\Omega} \oint_{r} \xi d x\right\},
\end{aligned}
$$

式中 $\Gamma$ 为包围 $Q$ 的边界，线积分取向应使 $Q$ 保持在左边; 〈〉的下标 $Q$ 表示在海区 $Q$ 上取平均; $\Omega$ 也表示该海区的面积.

\section{4. 方法 3 用上标 $*$ 表示一个复数的共轭,作运算}

$$
\begin{gathered}
\iint_{\Omega} \frac{1}{4}\left[h\langle\tilde{u}\rangle_{x}^{*} \cdot(4)+h\langle\tilde{u}\rangle_{x} \cdot(4)^{*}+h\langle\tilde{v}\rangle_{z}^{*} \cdot(5)+h\langle\tilde{v}\rangle_{z} \cdot(5)^{*}\right. \\
\left.+g \tilde{\zeta}^{*} \cdot(6)+g \tilde{\zeta} \cdot(6)^{*}\right] d \Omega,
\end{gathered}
$$

并应用 Gauss 公式,可得

$$
\iint_{0} \frac{1}{2} \operatorname{Re}\left[\tilde{\tau}_{x b}\langle\tilde{u}\rangle_{z}^{*}+\tilde{\tau}_{y b}\langle\tilde{v}\rangle_{x}^{*}\right] d S=-\int_{r^{\prime}} \frac{1}{2} g \operatorname{Re}\left[h \tilde{\zeta}\left\langle\tilde{u}_{n}\right\rangle_{z}^{*}\right] d l,
$$

式中 $u_{n}$ 为流速在 $\Omega$ 边界 $\Gamma$ 外法线上的射影, $F^{\prime}$ 为 $\Gamma$ 的开边界部分. 本式推导中已利用了在 $\Gamma$ 的闭边界上 $u_{n}=0$ 的条件.

虽然对像杭州湾这样的浅海,引潮力是小量, 但有时出于理论上的兴趣, 也希望估算一下 引潮力作的功. 这时方程 (11) 右端可加上引潮力作功的项.

单纯由式(11)不足以把包含 4 个未知量的 $\left(\tilde{\tau}_{x b}, \tilde{\tau}_{y b}\right)$ 解算出来, 这时必须引进海底应力 和流速之间的某种事先假定的关系，以使得未知量减少至 1 个.

\section{二、计 萁结果}

1. 方法 1 在杭州湾内分布着许多岛屿. 以这些岛屿为端点, 我们取了 5 个断面, 如图 $1 \mathrm{a}$ 所示. 这里各断面大体上沿着潮流的主流方向, $x^{\prime}$ 轴由湾内指向湾外. 根据式(8)算得的 角频率为 $\sigma_{M_{2}}$ 的海底应力 $\tilde{\tau}_{x^{\prime} b}$ 如表 1 所示.

在水力学中,常常采用底应力比例于流速平方的关系,写作

$$
\left(\tau_{x b}, \tau_{y b}\right)=k\left[\langle u\rangle_{z}^{2}+\langle v\rangle_{z}^{2}\right]^{1 / 2}\left(\langle u\rangle_{z},\langle v\rangle_{s}\right),
$$

$k$ 称为拖电系数. 但实际上对潮流这种关系并不一定很好满足. 首先 $x$ 和 $y$ 方向的 $k$ 值并不 一定相等, 其次底应力不一定在垂直平均潮流最强时达到最大. 作为式(12)的推广, 我们以 $\tilde{a}$ 和 $\tilde{b}$ 表示 $\left[\langle u\rangle_{z}^{2}+\langle v\rangle_{x}^{2}\right]^{1 / 2}\langle u\rangle_{x}$ 和 $\left[\langle u\rangle_{z}^{2}+\langle v\rangle_{z}^{2}\right]^{1 / 2}\langle v\rangle_{z}$ 的广义 Fourier 变换, 即

$$
(\tilde{a}, \tilde{b}) \equiv \frac{1}{T} \int_{-T}^{T}\left[\langle u\rangle_{s}^{2}+\langle v\rangle_{z}^{2}\right]^{1 / 2}\left(\langle u\rangle_{z},\langle v\rangle_{s}\right) d t,
$$

则底应力和流速的关系可表示为

$$
\left(\tilde{\tau}_{x b}, \tilde{\tau}_{y b}\right)=\left(\tilde{k}_{x} \tilde{a}, \tilde{k}_{y} \tilde{b}\right),
$$

这里 $\bar{k}$ 的模值代表了拖电系数的大小, 幅角代表底应力和潮流的位相差, 正角表示应力落后于 潮流，负角表示提前. 根据式(13)和(14)算得各断面的拖曳系数值亦列入表 1 , 表中 $k$ 和 $\theta$ 分 别为 $\tilde{k}$ 的模值和幅角. 从表 1 可看到各断面 $k$ 值很相近, $\theta$ 值有点分散, 但平均接近 $0^{\circ}$. 这 里取平均时以断面长度 $L$ 为权系数进行加权平均. 


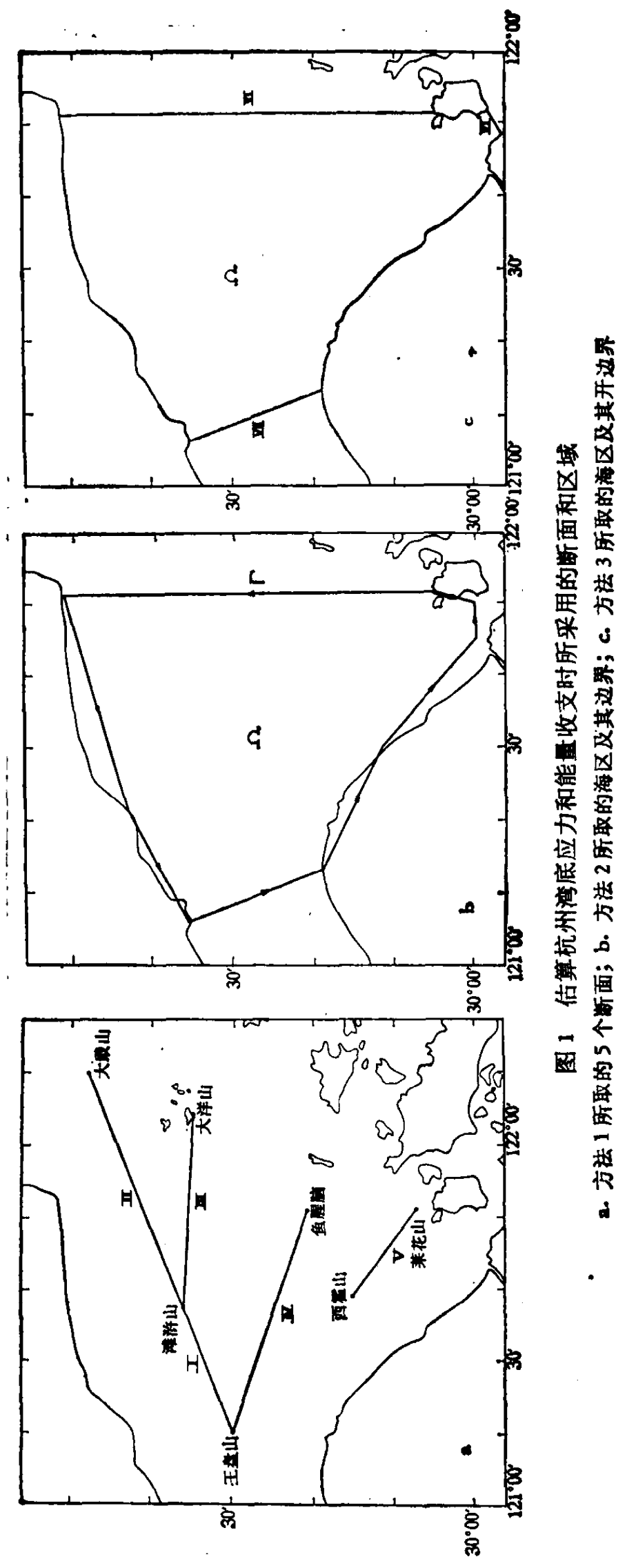


表 1 方法 1 估算所得的底应力及拖曳系数

\begin{tabular}{|c|c|c|c|c|c|}
\hline 断面号 & $\underset{m}{\langle h\rangle_{\boldsymbol{x}^{\prime}}}$ & $\begin{array}{r}\left\langle\tilde{\tau}_{x^{\prime} b}\right\rangle_{x^{\prime}} \\
10^{-4} \mathrm{~m}^{2} / \mathrm{s}^{2}\end{array}$ & $\begin{array}{c}\left\langle\tilde{k}_{x^{\prime}}\right\rangle_{x^{\prime}} \\
10^{-3}\end{array}$ & $\begin{array}{l}\left\langle k_{x^{\prime}}\right\rangle_{x^{\prime}} \\
10^{-3}\end{array}$ & $\begin{array}{c}\left\langle\theta_{x^{\prime}}\right\rangle_{\mathbf{x}^{\prime}} \\
\mathrm{deg}\end{array}$ \\
\hline $\mathbf{I}$ & 11.2 & $-7.29+i 4.56$ & $0.66+i 0.30$ & 0.72 & 24 \\
\hline II & 9.9 & $0.78+i 6.48$ & $0.64-i 0.22$ & 0.68 & -19 \\
\hline III & 10.2 & $0.24+i 8.47$ & $0.59-i 0.20$ & 0.62 & -19 \\
\hline IV & 11.8 & $-6.81+i 6.34$ & $0.60+i 0.27$ & 0.66 & 24 \\
\hline $\mathbf{v}$ & 10.4 & $3.48+i 5.40$ & $0.54-i 0.44$ & 0.70 & -39 \\
\hline & & & \multicolumn{2}{|c|}{ 加权平均: 0.67} & -4 \\
\hline
\end{tabular}

2. 方法 2 用方法 2 计算时我们取 $x$ 和 $y$ 轴分别指向东和北. 计算区域 $Q$ 及其边界 $\Gamma$ 如图 $1 \mathrm{~b}$ 所示. 所得结果见表 2 .

表 2 方法 2 估算所得的底应力及拖电系数

\begin{tabular}{c|c|c|c|c}
\hline 分 & $\begin{array}{c}\left\langle\tilde{\tau}_{b}\right\rangle_{0} \\
10^{-4} \mathrm{~m}^{2} / \mathrm{s}^{2}\end{array}$ & $\begin{array}{c}\langle\tilde{k}\rangle_{0} \\
10^{-3}\end{array}$ & $\begin{array}{c}\langle k\rangle_{0} \\
10^{-3}\end{array}$ & $\begin{array}{c}\langle\theta\rangle_{0} \\
\operatorname{deg}\end{array}$ \\
\hline$x$ & $-3.52+i 4.66$ & $0.49+i 0.11$ & 0.50 & 12 \\
$y$ & $0.66-i 4.93$ & $1.53-i 0.55$ & 1.63 & -20 \\
\hline
\end{tabular}

由表 2 看到, $y$ 方向的 $k$ 值明显地比 $x$ 方向大. 这样看来底摩擦并不是各向同性的. 但 是应当注意到, 杭州湾潮流主要是沿 $\boldsymbol{x}$ 方向, 且南岸潮汐观测资料不充分, 故 $y$ 方向的应力估 计值可能不会很准确,进一步观测和计算仍然是需要的.

3. 方法 3 在应用式(11)时, $\Gamma^{\prime}$ 由图 $1 \mathrm{c}$ 中断面 VI, VI' 和 VII 组成, $Q$ 为由 $\Gamma^{\prime}$ 和海 岸所包围的区域,计算结果见表 3 .

表 3 杭州湾 $\mathbf{M}_{2}$ 湖能收支

\begin{tabular}{l|c} 
通过断面 VI 连入的能通量 & $3.45 \times 10^{6} \mathrm{~kW}$ \\
通过断面 VI' 进入的能通量 & $0.19 \times 10^{6} \mathrm{~kW}$ \\
通过断面 VII 出去的能通 & $1.29 \times 10^{6} \mathrm{~kW}$ \\
单位时间引湖力所作的功 & $-0.017 \times 10^{6} \mathrm{~kW}$ \\
本海区单位时间消耗的潮能 & $2.33 \times 10^{6} \mathrm{~kW}$
\end{tabular}

如上所述,单纯由式(11)不能直接得出包含 4 个未知量的底应力 $\left(\tilde{\tau}_{x b}, \tilde{\tau}_{y b}\right)$ ，因而也不能 由式(14)计算 $\left(\tilde{k}_{x}, \tilde{k}_{y}\right)$. 这时只能利用假设 (12). 由此算得海区内平均的〈k $\langle$ 。值为 $0.54 \times$ $10^{-3}$, 与由方法 2 得出的 $\left\langle k_{x}\right\rangle_{0}$ 颇接近.

4. 结语 上述方法及所用资料具有一定相互独立性, 而所得拖电系数较一致，在 $0.5-$ $0.67 \times 10^{-3}$ 之间. 这说明结果是反映客观实际的. 拖曳系数数值只有国外一般采用数值的 $1 / 3-1 / 4$, 这对杭州湾动力学研究和数值计算具有重要意义,也表明杭州湾潮流中的湍流结构 值得进一步深人观测和研究. 横向拖曳系数显著大于纵向的现象也值得进一步检验和研究。

\section{考文嗝}

[1 ] Bowden, K. F. and Fairbairn, L. A., Proc. Roy. Soc., A214 (1952), 371-392.

[ 2 ] Wolf, J., in Marine Turbulence (Ed. Nihoul, J. C. J.), Elsevier Sci. Publ. Co., 1980, 319-344.

[ 3 ] Tyylor, G. I., Phil. Trans. Roy. Soc., A220(1919), 1-93. 